\title{
Structure and mechanical properties of aluminum - aluminum nanoparticles composite produced by accumulative roll bonding
}

\author{
K.V. Ivanov ${ }^{\dagger}$, N.G. Rodkevich, S.V. Fortuna, T.A. Kalashnikova \\ †ikv@ispms.tsc.ru
}

Institute of Strength Physics and Materials Science of Siberian Branch of RAS, 2/4 Akademichesky Av., 634055, Tomsk, Russia

A modified accumulative roll bonding (ARB) process was used to obtain ultrafine-grained aluminum matrix composite with aluminum nanoparticles produced by electric wire explosion and inserted between the bonded stripes. On the preliminary stage the pack consisting of two $1 \mathrm{~mm}$ thick stripes and nanopowder between them was rolled up to a significant strain level of $85 \%$ to obtain the well bonded thin stripe. On the first main stage (the first ARB cycle) the pack consisting of two ordinary stripes and a thin one with nanoparticles was rolled and cut in half. The sheet of Al-1\%nAl composite was obtained as a result of $4 \mathrm{ARB}$ cycles. For a comparison, commercial aluminum was processed under the same route without nanopowder insertion. Formation of an ultrafine-grained structure in both materials was found. Nanoparticles formed totally compacted layers between the bonded surfaces. The thickness of the layers decreased with the increase of the number of cycles, while the size of the structure elements remained unchanged. In the areas corresponding to the thin stripe inserted at final cycles of ARB, a decreased microhardness was observed owing to the less accumulated strain as compared to the ordinary stripes. The reasons for the weak effect of aluminum nanoparticles on the structure and tensile strength of the rolled products are discussed.

Keywords: accumulative roll bonding, metal matrix composites, nanopowders, ultrafine-grained structure.

\section{Структура и механические свойства композита}

\section{алюминий - наночастицы алюминия,}

\section{полученного многократной прокаткой с сопряжением слоев}

\author{
Иванов К.В. ${ }^{\dagger}$ Родкевич Н.Г., Фортуна С.В., Калашникова Т.А. \\ Институт физики прочности и материаловедения СО РАН, пр. Академический 2/4, 634055, Томск, Россия
}

\begin{abstract}
Для формирования ультрамелкозернистого композиционного материала с алюминиевой матрицей и наночастицами алюминия, полученными методом электрического взрыва проволоки, введенными между сопрягаемыми пластинами, использован модифицированный способ многократной прокатки с сопряжением слоев. На предварительном этапе прокатывали пакет, состоящий из двух пластин толщиной 1 мм, с нанесенным нанопорошком на сопрягаемых поверхностях до значительной степени деформации (85\%) для того, чтобы получить тонкую пластину-вставку с хорошим сопряжением слоев. На первом основном этапе (первом цикле многократной прокатки с сопряжением слоев) формировали пакет из обычных пластин с помещением между ними тонкой пластины - вставки с наночастицами, прокатывали этот пакет и разрезали пополам. В результате 4 циклов прокатки была получена полоса композита $\mathrm{Al}-1 \% \mathrm{nAl}$. Для выяснения роли наночастиц в формировании структуры и свойств композита таким же способом получали материал без введения нанопорошка. Обнаружено, что в результате описанной обработки в обоих материалах формируется ультрамелкозернистая структура. Наночастицы образовывали полностью компактированные слои между сопрягаемыми пластинами. Толщина этих слоев уменьшалась с увеличением числа циклов прокатки, а размер элементов структуры в них оставался неизменным. В областях материала, соответствующих пластине вставке, введенной на заключительных циклах прокатки, значение микротвердости снижается вследствие меньшей степени деформации, накопленной при обработке. Обсуждаются возможные причины незначительного влияния наночастиц алюминия на формирование структуры и механических свойств при испытаниях на растяжение композиционного материала
\end{abstract}

Ключевые слова: многократная прокатка с сопряжением слоев, композиционные материалы с металлической матрицей, нанопорошки, ультрамелкозернистая структура. 


\section{1. Введение}

В последние годы интерес к композиционным материалам с металлической матрицей для изготовления ответственных изделий неуклонно растет благодаря тому, что они обладают привлекательным сочетанием свойств: высокой термической и электрической проводимостью, низким коэффициентом термического расширения, высоким отношением прочности к удельному весу, значительной износостойкостью и другими.

Актуальными, с точки зрения создания новых композиционных материалов с улучшенными свойствами, являются исследования по разработке и созданию композитов, в которых наряду с упрочнением дисперсными частицами реализуют структурное упрочнение за счет формирования ультрамелкозернистого состояния в матрице путем интенсивной пластической деформации. Традиционно формирование ультрамелкозернистых композитов с металлической матрицей включает два этапа: на первом формируется собственно композит (методами порошковой металлургии или внутреннего окисления), на втором полученная заготовка подвергается воздействию интенсивной пластической деформации с целью измельчения зеренной структуры $[1,2]$. Данный подход внутренне противоречив, так как интенсивной деформации подвергается заготовка композита, которая уже упрочнена за счет введения частиц, поэтому процесс деформации и измельчения зерна матрицы затруднен. Перспективным представляется подход, при котором введение частиц в материал матрицы происходит непосредственно в процессе интенсивной пластической деформации, например, способом многократной прокатки с сопряжением слоев (в англоязычной литератуpe accumulative roll bonding - ARB). Частицы наносятся на сопрягаемые поверхности перед заданным циклом прокатки [3-5]. При использовании этого способа есть возможность в широких пределах варьировать тип частиц, изменять их объемную долю и области внесения. Способ обладает теми преимуществами, что реализуется на стандартном деформационном оборудовании (прокатные станы), не требует сложной технологической оснастки, использует стандартное сырье в виде технически чистых металлов и имеющихся на рынке ультрадисперсных порошков, а получаемый материал имеет удобную форму металлопроката. Указанные достоинства открывают перспективы для практического применения ультрамелкозернистых композитов, полученных способом многократной прокатки с сопряжением. Обычно в качестве упрочняющей фазы используются порошки оксида алюминия или карбида кремния с микронным размером. Применяется также способ нанесения покрытий или химического преобразования материала поверхности заготовок [3-5]. Известно, что уменьшение размера частиц второй фазы в композиционных материалах с металлической матрицей дает возможность снизить долю этой фазы с одновременным улучшением свойств - увеличением прочности, снижением электросопротивления и других. Поэтому перспективно использование порошков с наноразмерными частицами.
В связи с вышеизложенным в работе была поставлена задача получить ультрамелкозернистый композиционный материал на основе алюминия со второй фазой в виде частиц размером менее 100 нм методом многократной прокатки с сопряжением слоев и исследовать влияние частиц второй фазы на формирование структуры и механических свойств материала при указанной обработке.

\section{2. Методика эксперимента}

В качестве исходного материала использовали алюминий марки А7 (по ГОСТ 11069 - 2001). Полосы размером $250 \times 30 \times 1$ мм $^{3}$ перед прокаткой отжигали при температуре 773 К в течение 7200 c.

Наиболее простой и часто используемый способ многократной прокатки с сопряжением слоев заключается в следующем. Материал в виде двух полос толщиной 1 мм подвергают поверхностной обработке металлической щеткой. Затем из них формируют пакет, который прокатывают до толщины 1 мм, то есть на 50\%. Полученная полоса разрезается пополам. Указанная последовательность действий рассматривается как один цикл многократной прокатки. Циклы повторяют несколько раз до достижения заданной степени деформации [6]. Степень эквивалентной истинной деформации за один цикл прокатки равна 0,8 .

В настоящей работе для процесса многократной прокатки с сопряжением слоев при комнатной температуре использовали прокатный стан ВЭМ-3 с диаметром валков 80 мм и скоростью прокатки 0,1 м/с. Поверхностную обработку проводили стальной щеткой с диаметром проволоки 0,3 мм на токарном станке. В качестве модельной дисперсной фазы использовали нанопорошок алюминия с размером частиц 50 нм, полученный методом электрического взрыва проволоки в лаборатории физикохимии высокодисперсных материалов ИФПМ CO PAH [7]. Обозначим эту фазу как nAl. Несмотря на то, что в качестве второй фазы использовали то же вещество, что и материал матрицы, ожидали, что вторая фаза окажет влияние на свойства композиционного материала, так как наночастицы алюминия подвергали поверхностному окислению, в результате чего массовая доля оксида алюминия на поверхности частиц равнялась $10 \%$ [8], и влияние должно было быть обусловлено наличием оксида алюминия и наноразмером дисперсной фазы. Массовая доля порошка, внедряемого в материал, составляла $1 \%$.

Для нанесения второй фазы на сопрягаемые поверхности необходимое количество нанопорошка помещали в ацетон, после чего подвергали воздействию ультразвука в течение 600 с. Взвесь нанопорошка в ацетоне наносили на подготовленные поверхности полос, затем выдерживали до полного испарения ацетона. При прокатке пакета с нанопорошком на 50\% сцепления пластин не достигается. Для размера пластин и массовой доли наночастиц, использованных в эксперименте, сцепление происходит при степени деформации пакета выше 70\%. Однако в этом случае при последующих циклах прокатки нарушается постоянство толщины заготовки (тол- 
щина уменьшается с каждым последующим циклом), а способность подвергать материал экстремально большим степеням деформации без значительного изменения геометрических размеров заготовки является одним из достоинств метода интенсивной пластической деформации [9]. В связи с этим обычный способ многократной прокатки с сопряжением был модифицирован. На предварительном этапе брали две полосы, подвергали вышеуказанной поверхностной обработке и на подготовленные поверхности наносили нанопорошок, после чего формировали пакет и прокатывали на $85 \%$. В результате получали полосу-вставку толщиной 0,3 мм, содержащую на внутренней поверхности сопряжения наночастицы алюминия. Эту полосу разрезали на части длиной, соответствующей длине исходных пластин. На первом основном этапе формировали пакет из трех пластин двух внешних, подготовленных обычным способом, и полосы-вставки, полученной на предварительном этапе. Пакет прокатывали с толщины 2,3 мм до толщины 1 мм, степень деформации за проход составляла 57\%, а эквивалентной истинной деформации 1,0 . Полученную пластину разрезали пополам. Описанные действия рассматривали как один цикл модифицированной многократной прокатки с сопряжением. Был получен композиционный материал Al-1\%nAl, подвергнутый 4 циклам прокатки. Для выяснения влияния второй фазы на формирование структуры и свойства был получен контрольный образец по такому же режиму, но не содержащий нанопорошка в пластине-вставке.

Структуру исследовали методом просвечивающей электронной микроскопии в продольном вертикальном сечении. Фольги готовили методом ионного утонения на установке EM-09100IS фирмы Jeol при ускоряющем напряжении 6 кВ и угле падения ионного пучка $4^{\circ}$. Исследования проводили с использованием микроскопа JEM-2100 при ускоряющем напряжении 200 кВ.

Микротвердость материала по методу Виккерса измеряли на приборе Duramin-5 при нагрузке на индентор $0,25 \mathrm{H}$ в течение 15 секунд.

Растяжение образцов с размером рабочей части $20 \times 6,5 \times 1$ мм $^{3}$ проводили на испытательной машине INSTRON 3369 при комнатной температуре со скоростью $1 \times 10^{-4} \mathrm{c}^{-1}$.

\section{3. Результаты эксперимента и их обсуждение}

Электронномикроскопические исследования показали, что в композиционном материале Al-1\%nAl, полученном методом многократной прокатки с сопряжением слоев, сформирована ультрамелкозернистая структура с размером элементов зеренно-субзеренной структуры в интервалах 120 - 500 и 1200 - 3000 нм поперек и вдоль направления прокатки, соответственно (рис. 1). Следует отметить, что при обычных условиях прокатки технически чистого алюминия такая степень измельчения элементов структуры достигается при большем числе циклов обработки [10]. Последнее связано, по-видимому, с тем, что при использованном в работе модифицированном способе многократной прокатки пакет подвергается большей степени деформации за один цикл по сравнению со стандартным способом ( 1,0 вместо 0,8$)$, и образуется большее число поверхностей сопряжений (45 за 4 цикла вместо 31). Небольшая часть элементов зеренно-субзеренной структуры имеет равноосную форму, не содержит дислокаций и окружена прямыми границами с полосчатым контрастом на них. Можно полагать, что эти элементы являются зернами, сформировавшимися в результате динамической рекристаллизации. На рис. 1а черными стрелками отмечен след поверхности сопряжения, первоначально содержащей наночастицы. Видно, что элементы зеренно-субзеренной структуры, лежащие по обе стороны этой поверхности, имеют поперечный размер 50 - 70 нм, что совпадает с диаметром частиц нанопорошка алюминия, внедренного в поверхность раздела. Таким образом, в процессе многократной прокатки с сопряжением нанопорошок алюминия компактируется и образует сплошной массив материала, не содержащий пор. Сопротивление миграции границ внутри такого массива существенно выше, чем в основном материале из-за того, что на поверхности нанопорошка алюминия всегда присутствует оксидная пленка, служащая препятствием для движения границ.

Приведенные выше суждения хорошо подтверждаются изображением области, материал в которой образован скоплением нанопорошка алюминия между поверхностью раздела, полученной, вероятно, в результате заключительного цикла прокатки (рис. 1b). В областях скопления порошка образуется сплошной массив практически беспористого материала, поры располагаются только на границе массив порошка - основной материал. Видно, что размер элементов структуры в этих областях не превышает 100 нм. Из-за того, что свойства нанопорошка алюминия позволяют вносить его в довольно большой концентрации при прокатке с сопряжением, размер областей скопления нанопорошка внутри материала может достигать значительного размера - до нескольких десятков микрон (рис. 1b). В результате последующих циклов прокатки этот размер уменьшается.

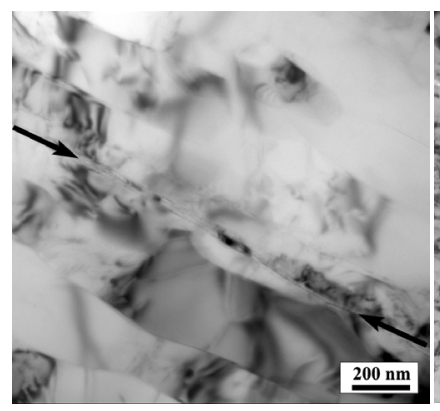

a

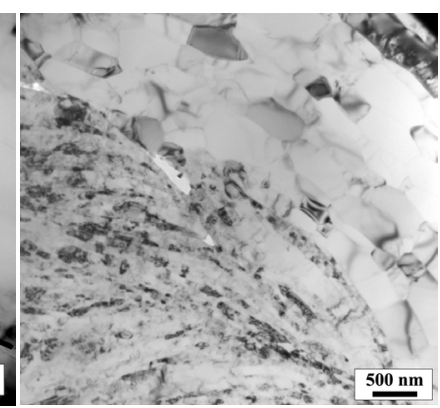

$\mathrm{b}$
Рис. 1. Структура композита Al-1\%nAl. Стрелками указан слой компакта из нанопорошка алюминия в полосе-вставке, внедренной на начальном цикле прокатки (a). $\mathrm{Ha}$ (b) в левой нижней части видна протяженная область компакта из нанопорошка алюминия, внедренного на заключительном цикле прокатки.

Fig. 1. Structure of $\mathrm{Al}-1 \% \mathrm{nAl}$ composite. Black arrows indicate the compact in the stripe inserted on early stages of ARB (a). One can see the extended area of aluminum nanopowder compact injected on the final cycle of ARB in the left bottom corner of (b). 
Следует отметить, что в области, соответствующей введенной на последних циклах прокатки пластине с наночастицами, размер элементов зеренно-субзеренной структуры алюминиевой матрицы значительно выше, чем в основном объеме материала (до нескольких микрон), а структура разбивается на субзерна с малоугловыми границами и дислокационными образованиями. Такая структура характерна для материалов, деформированных на относительно небольшие степени деформации по сравнению с величинами, достигаемыми при интенсивной пластической деформации.

Изменение величины микротвердости в зависимости от расстояния от поверхности вдоль направления, нормальному направлению прокатки, для композита Al-1\%nAl и контрольного образца технически чистого алюминия приведено на рис. 2. Видно, что для обоих материалов поведение микротвердости подобно: максимальные значения зафиксированы вблизи внешних поверхностей заготовки и на расстояниях от них, соответствующих трети толщины заготовок. На зависимостях имеются и минимумы в районе середины заготовок и на расстояниях от поверхности, соответствующих четверти их толщины. Очевидно, что минимальные значения микротвердости соответствуют областям расположения полосы-вставки. Причем в середине, где вставка подвергнута лишь однократной прокатке в пакете в результате последнего цикла многократной прокатки, значение микротвердости ниже, чем в областях 0,25 и 0,75 мм, соответствующих положению вставки после двух последних циклов прокатки. Отметим большой разброс значений микротвердости в середине заготовки. Он связан, вероятно, с тем, что в этих областях укол индентора может попадать как в полосу-вставку, так и в граничащую с ней полосу с большей степенью накопленной деформации.

Испытания на растяжение показали, что прочностные свойства полученного композита незначительно превышают свойства технически чистого алюминия после соответствующей обработки, также как и удлинение до разрушения (табл. 1, рис. 3). Слабо выраженное влияние наночастиц на свойства композита может быть связано с двумя факторами: во-первых, наночастицы локализованы на поверхностях сопряжения. Число поверхностей сопряжения с локализованными на них наночастицами при числе циклов прокатки составляет 15 , следовательно, в полосе толщиной 1 мм они расположены на расстоянии около 65 мкм. Понятно, что в этом случае основной объем материала практически не подвержен их влиянию. Очевидно, что для усиления роли наночастиц в формировании структуры и свойств композита необходимо более равномерное их распределение в объеме материала. Этого можно добиться путем увеличения числа циклов прокатки. Во-вторых, материал матрицы и наночастиц один и тот же. Разница лишь в наличии на поверхности наночастиц оксидного слоя, который, собственно, и должен влиять на свойства получаемого композита.

Подобный вид профилей микротвердости, полученных для композиционного материала Al-1\%nAl и технически чистого алюминия, близость их прочностных свойств и величин деформации до разрушения при испытаниях на растяжение указывает на принципиальную возможность внесения второй фазы в виде наноразмерного порошка при многократной прокатке с сопряжением слоев без снижения механических свойств за счет формирования несплошностей и микротрещин.

\section{4. Выводы}

1. Показано, что модифицированный способ многократной прокатки с сопряжением слоев позволяет формировать ультрамелкозернистый композиционный материал на основе алюминия с внедрением в качестве второй фазы наночастиц алюминия без ухудшения механических свойств по сравнению с алюминием без наночастиц.

2. Установлено, что при многократной прокатке с сопряжением слоев наночастицы алюминия образуют компактированные прослойки вдоль поверхностей сопряжения без пор и микротрещин. С увеличением

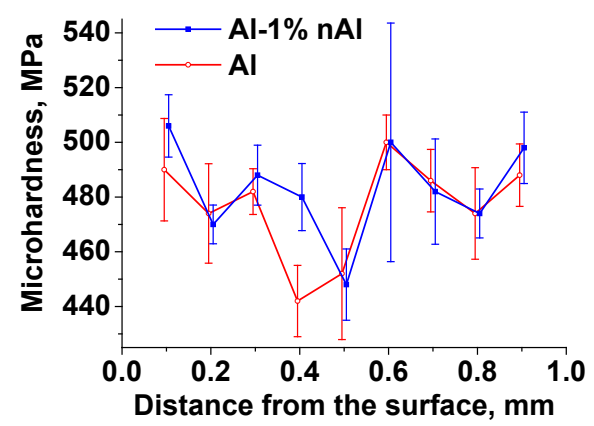

Рис. 2. Профиль микротвердости вдоль направления, нормальному направлению прокатки.

Fig. 2. Microhardness profile along the normal direction.

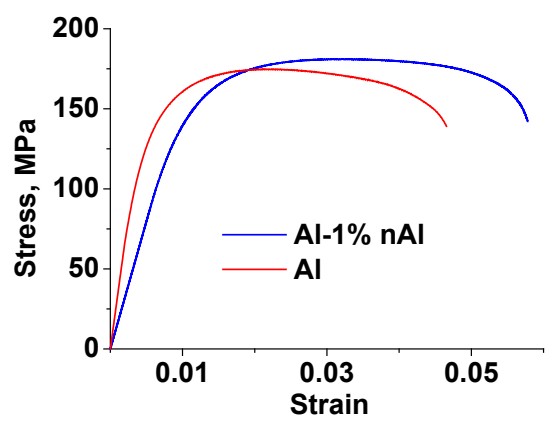

Рис. 3. Кривые течения композита и алюминия.

Fig. 3. Flow curves obtained for the composite and aluminum.

Табл. 1.Предел прочности $\left(\sigma_{\mathrm{B}}\right)$, предел текучести $\left(\sigma_{02}\right)$ и удлинение до разрушения $(\delta)$ композита $\mathrm{Al}-1 \% \mathrm{nAl}$ и технически чистого алюминия.

Table 1. Ultimate strength $\left(\sigma_{\mathrm{u}}\right)$, yield strength $\left(\sigma_{\mathrm{y}}\right)$ and elongation up to failure $(\delta)$ of Al-1\%nAl composite and aluminum.

\begin{tabular}{|c|c|c|c|}
\hline $\begin{array}{c}\text { Материал } \\
\text { Material }\end{array}$ & $\begin{array}{c}\sigma_{\mathrm{B}}, \mathrm{MПа} \\
\sigma_{u}, \mathrm{MPa}\end{array}$ & $\begin{array}{c}\sigma_{02}, \mathrm{MПа} \\
\sigma_{y}, \mathrm{MPa}\end{array}$ & $\begin{array}{c}\delta, \% \\
\delta, \%\end{array}$ \\
\hline $\mathrm{Al}-1 \% \mathrm{nAl}$ & 181 & 152 & 4.7 \\
\hline $\mathrm{Al}$ & 175 & 144 & 4.2 \\
\hline
\end{tabular}


степени накопленной деформации их толщина уменьшается вплоть до величины, соответствующей размеру наночастиц. Размер элементов структуры в прослойках не изменяется при деформации вследствие наличия на поверхности частиц оксидного слоя, эффективно тормозящего процесс динамической рекристаллизации.

3. Обнаружено, что композит имеет неоднородную структуру и микротвердость по толщине заготовки, что связано с различной степенью накопленной деформации разных слоев при многократной прокатке с сопряжением.

4. Влияние наночастиц на структуру и свойства композиционного материала незначительно вследствие локализации наночастиц по поверхностям раздела, разделенными в материале значительными расстояниями, а также того, что матрица и наночастицы состоят из одного и того же вещества.

Благодарность/Acknowledgements. Исследование выполнено при финансовой поддержке РФФИ и Администрации Томской области в рамках научного проекта № 16-43-700440. Структурные исследования методом просвечивающей электронной микроскопии проведень на оборудовании центра коллективного пользования "Нанотех» в ИФПМ СО РАН, механические испьтания - на оборудовании Томского материаловедческого иентра коллективного пользования Национального исследовательского Томского государственного университета.

\section{Литература/References}

1. R.K. Islamgaliev, W. Buchgraber, Yu. R. Kolobov, et al. Mater. Sci. Eng. 319-321A, $872-876$ (2001), DOI: 10.1016/S0921-5093 (01) 01073-5

2. K. V. Ivanov, G.P. Grabovetsaya, Yu. R. Kolobov, et al. Adv. Mater. 4, 78 - 84 (2001). (in Russian) [К. В. Иванов, Г. П. Грабовецкая, Ю. Р. Колобов, и др. Перспективные материалы 4, 78 - 84 (2001).]

3. R. Jamaati, M.R. Toroghinejad, A. Najafizadeh. Mater. Sci. Eng. 527, $3857-3863$ (2010), DOI: 10.1016/j. msea.2010.08.038

4. R. Jamaati, S. Amirkhanlou,, M. R. Toroghinejad, B. Niroumand. Mater. Sci. Eng. 528, 2143 -2148 (2011), DOI:10.1016/j.msea.2010.11.056

5. S. V. A. Ana, M. Reihanian, B. Lotfi. Mater. Sci. Eng. 647, 303 - 312 (2015), DOI:10.1016/j.msea.2015.09.006

6. Y. Saito, N. Tsuji, H. Utsunomiya, T. Sakai, R. G. Hong. Scripta Mater. 39, $1221-1224$ (1998), DOI: 10.1016/S1359-6462 (98) 00302-9

7. M. I. Lerner, N. V. Svarovskaya, S. G. Psakhie, O. V. Bakina. Nanotechnologies in Russia. 4 (11-12), 741 - 757 (2009), DOI: $10.1134 /$ S1995078009110019

8. M. I. Lerner, V. V. Shimanskiy, G. G. Saveliev. Bulletin of the Tomsk Polytechnic University. 310 (2) 122 - 126 (2007).

9. R.Z. Valiev, R. K. Islamgaliev, I. V. Alexandrov. Prog. Mater. Sci. 45, $103-189$ (2000), DOI: 10.1016/S00796425 (99) $00007-9$

10. K. V. Ivanov. AIP Conference Proceedings 1783, 020076 (2016), DOI: 10.1063/1.4966369 